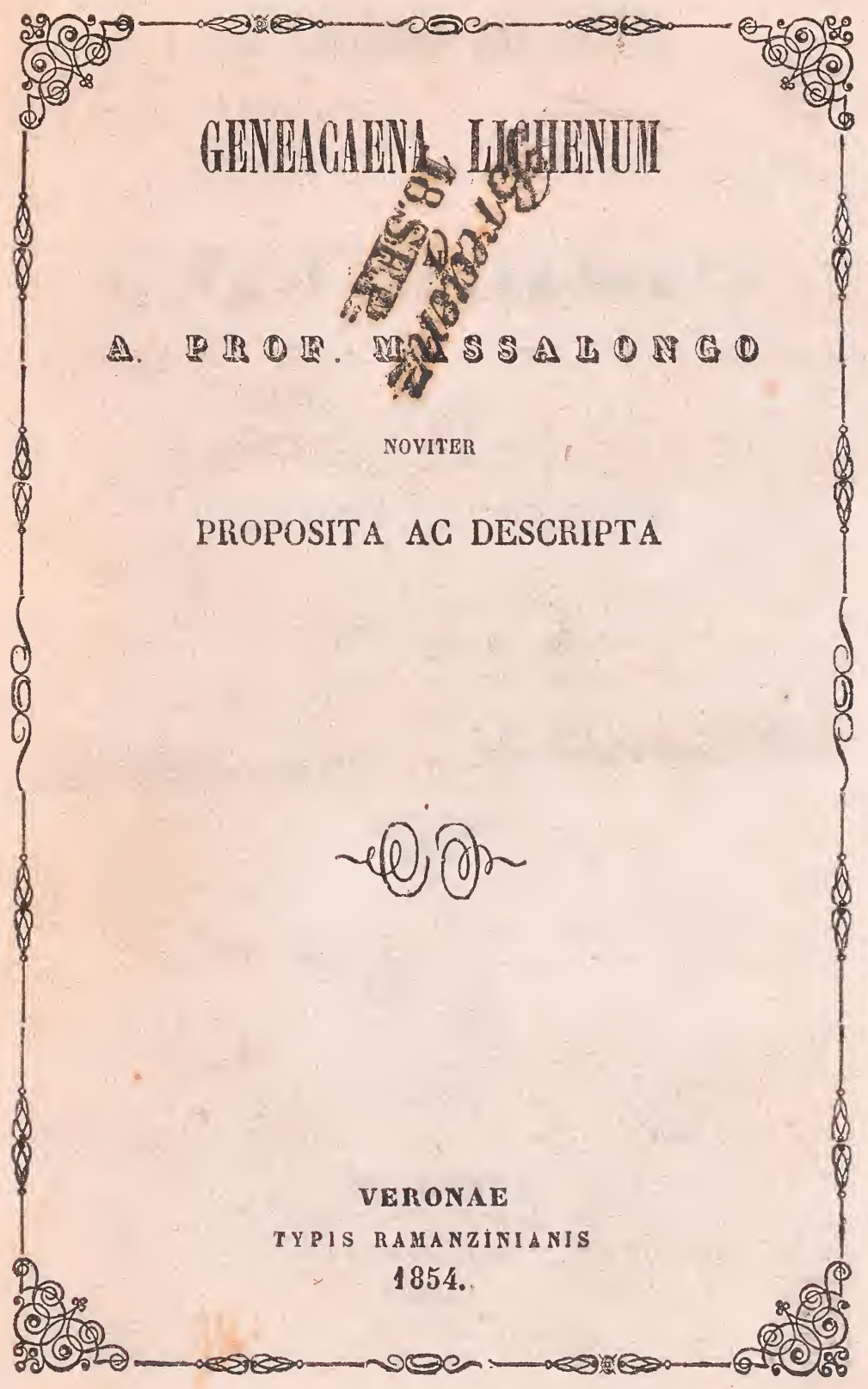





\section{GENEAGAENI LCGHENOII}

$A B$

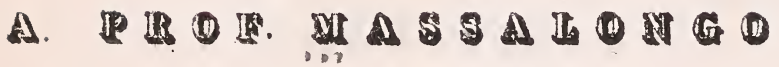

NOVITER

PROPOSITA AC DESCRIPTA

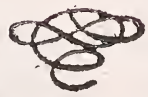

LIBRARY

DESW YORK

"MT"ANYCAL

A

\section{VERONAE}

TYPIS RAMANZINIANIS

1854. 
QK584

. $M 38$ 


\title{
(क) (क) (क)
}

\author{
LECTORI
}

In animo milii est hic breviter describere quae. dam lichenum genera, quorum alia nunc primum a me proponuntur, alia vero a me ipso ad arctiores limites praefiniuntur; perinde ac si satis haud essent nova illa, quae in lichenologiam immisi, nec satis superque sint jam antea statuta. - Hinc mihi ultro veniam, peto, praebeant lichenologi, iique in primis qui mecum non conveniunt. In methodo quam amplexus sum magis thagisque confirmor; eamque recto tramite sequor, pro certo habens, eos quoque vel cito vel serius in meam sententiam abituros.

Illud tantum mea interest, ut lichenologiae culto. res sibi persuadeant, me hoc non agere novitalis causa, nec inani cupiditate impulsus, ut aliis praecellam: nec tribuant quod perago ac peregi levitati mentis, aut puerili jactantiae: etenim si quo errore fortasse deceptus fuerim, ut plurimum tamen pro explorato haberi possunt ea quae scripsi; quaeque comnisi hujusmodi vitia sunt, quibus vix cavere humana 
natura potest, innituntur enim speciminibus illis, quae veluti authentica ab eximiis lichenologiae pa. rentibus in vulgus edita fuere.

Quomodocumque sit, mea collectio documento erit omnibus, quo mea defensio fulciatur. Diagrammata ac descriptiones cunctorum generum cunctarumque specierum, quas ibidem recensui, quamprimum pubblici juris fient, in singulari opusculo, statim ac negotia quibus distineor, et valetudo non satis firma, mihi concesserint. In quadam vero adnotatione posui (in finem hujus opusculi) enumeratio. nes aliquot specierum novarum, necnon emendationes quasdam, quae sunt faciendae meis operibus jam editis, quas debeo celeb. C. Montagne, et amicis praeclaris meis Körber et Krempellauber.

Ut mei moris est, quae scribo in nullum ordinem nullamque methodum redigo, et de hoc sese jac. tent cubicularii scriptores. 'Tantunmodo vero religiose servantur distinctiones Lichenum in Angiocarpos ac Gymnocarpos, quae prorsus sunt juxta naturalem philosophiam firmae et tutae, quid quid dicat aliquig bujus temporis scriptor. Vale.

Daban Veronar, M. Augnsti.

1854: 


\section{A Grunocarit}

1. KOERAERIA NOY. GEY.

$A_{\text {pothecia biatorina, primum punctiformia (fere clausa), }}$ dein plus minusve explanata patellaria, excipulo pseudo-thal lode in proprium mutato, albo-farinoso, instructa. Lamina pros ligera (carneo-miniata ) ceraceo-gelatinosa, tenerrima, hypothe cio crassiusculo cum excipulo connivente, imposita, licheninaque $\left(^{*}\right)$ praedistincta praedita. Asci crebri parvi 8-spori. paraphyssibus gelatinosis (apice fuscidulis). obvallati, sporidia $\mathrm{a}^{\mathrm{a}}$ ciformia, vermicularia, contorta, longa, filiformia, flexuosa, diaphana, homogenea s. unilocularia.

Thallus biformis, primum planiusculus platyphyllinus lobulato-cristatus ( supra laete virens, subtus albus), tandem isioi. deo-filameutoso-ramulosus, teres, undique concolor, e quatuor stratis distinctis constitutus: corticalis nempe e cellulis diaphanis pentagonis tetragonisque praedistinctis, hypothallinicuss (in statu platiphyllino) subspongiosus, e cellulis diaphanis serialibus praelongis, articulatis, s. parallelogrammaticis: mesothallinicus superior, e cellulis longissimis filamentosis inarticulatis diaphanis ramosis, mucilago achromatica obvolutis; inferior e $\mathrm{go}^{\circ}$ nidiis multiformibus in substantia mucilaginosa colorata nidu. lantibus, scilicet e coniogonidiis, diplogonidiis, ormogonidiis, gastrogonidiis, encatogonidiis croceis viridibusque, nec non idiosporis ferruginiis, compositus. Spermatocalia, tromodoblastia hucusque ignota.

(w) Lichenina est substantia isomerica cum amúlo, quaeque caerulen colore tingitur per Iodium. Reperitur in Muscis el Lichenibus, substantiae gelat nosae comixta. Ita ut guum dico Lamina proligera lichenina praedita, idem est as i Cl. Nylanderi verbis, dixerim: Mucilayo hymensa jodio cerulescin 
Observatio. Nova vocabula et ane parun euphonica quibus hoc loco utoz, albi explicabuntur, necessitas me compulit ut eadem efformarem. Dicatur boc gex nas amico preclaro D. G. Körber Vratizlaviessi, de recenti lichenologia optime merito.

\section{I) Koerberia biformis Nov. Spec.-}

Habit. ad troneos Populorum Veronénsium, in oppido vula go dicto Cucca. Legi.

II. COLLOLECIIA NOV. GEN.

Apothecia catothalama lecideina, semper aperta, primum punctiformia, dein patellaria plana, margine excipuloque proprio carbonaceo praecrasso praedita. Lamina proligera ceraceocornea, atra, licheninaque instructa, strato gonimico crasso imposita. Asci saccati 8-10-spori, paraphysibus crassis stipatis crassiusculis granuloso-articulatis, obvallati; sporidia costanter diaphana proteiformia-polymorpha, primum minutissime-subrotunda homogenea monolocularia, inde ovoidea-bilocularia, dein ellip. tica irregulariter granuloso-nucleifera, tandem (normaliter) baculiformia, contorta, 4-6-8-lucularia.

Thailus limitatus homaeomericus, crustosus, e cellulis gonimicis polymorphis, scil. e gonidiis, coniogonidiis (trichomatibus) encatogonidiisque croceis viridibusque in substantia gelatiirosa inordinate nidulantibus, compositus : centro e squamulis corallinoideis, bullosis, ambitu laciniato-effiguratis, hyphotallo spotgioso-fibrilloso, constitutus.

Obs. Genus affue Lecotheciis, tamen diversum ob thalli structuram, spora. rum apothecioruraque naturam. Permulta specimina authentica Lecideae caesia quae dissimili aetate accepi, in causa fuerunt propter quan tot judicia protuli de hos lichene. Qui penitius dabit operam hujus speclei, aequa animo indulgebit. 


\section{2) Collolechia caesia Massal.}

Synon. Racoblenna caesia Massal. Ricerch. pag. 140 fig. 275

(sporae juren.) - Racoblenna Garovagli Massal. Mem. lich. pag. 134 - Lecidea caecia Duf. Garov!! etc. -

\section{I. GUSSONEA TORNAB. (EMEND.)}

Apothecia patellaria primitus clausa, dein aperta, excipulo omnino thallode marginata. Lamina proligera thallo concoloria, hypothecio tenui imposita. Asci clavati 'monospori, paraphysitus apiee incrassatis, obvallati. Sporidia ovoideo-elleptica majuscula, tetrablassia diplopyrenia, subcolorata.

Thallus subfoliaceus crustaceusve effiguratus, radioso-plicatus, matricibus arcte adhaerens.

\section{3 ) Gussonea chlorophana Tornab.}

Lich. sicul. pag. 22, 1849 ( synon. Acarospora Massal. Ricerch. pag. 27 fig. 44 (protoblastia s. spororum initial)Lecanora Ach!

\section{4) Gussonea oxytona Massal.}

Syn. Acarospora Massal. loc. cit. pag. 28 fig. 45 (protoblastia ) Lecanora Ach!

IV. HEPPIA NAEG.

Apothecia in thalli pagina superiore sparsa, primum clausa, dein explanato patellaria, depresso-saccata, urceolata, in thalli pagina inferiore pustularum ad instar, protuberantia. (Veluti in salorina saccata!), excipulo destituta, margineque thallode ele. 
rato, undique cincta. Lamina protigera tenuis ceracea, lichenina destituta, hypothecioque crasso gonidiifero (non éxcipnlum!) imposita. Asci clavati 8-spori, paraphysibus stipatis crassiưsculis, obvallati; sporidia ovoidea oleoso-granulosa, diaphana, homo. genea.

Thallus ea subfoliosus matricibus arcte adhaerens s. adglutinatus, submonophyllus subimbricatus, e quatuor stratis constitutus: corticalis, e cellulis diaphamis irregularibus saepe obsoletis; hypothallinicus, spongiosus : mesothallinicus superior e chrisogonidiis latis, encatogonidiisque croceis in substantia granuloso-amylacea acromatica obrolutis; inferior e gastrogonidis magnis, subtilibusque encatogonidiis viridibus, compositus.

Obs. Genus meo quidem judicio distinctum, yuodque medlum tenet locum inter Parrizelias et Collemata.

\section{5 ) Heppia adglutinata Massal.}

Syn. Lecanora Kremp! in Flora Regnesa. Ratisb. 1851. Heppia urceolata Neg. Hepp. Lich. Gurop. n. 49 ! -

Y. CURMOTHECIUM NOV. GEN.

Apothecia catothalama s. ex hypothallo orinuda, siam primitus stipitata, ceqhaloidea glebosa, ezcipulo proprio carbonaceo atro in stipitem subattenuato, praedita. Lamina proligera Lichenina praedita, ceracea immarginata epithecio scabriusculo in testa, hypothecioque sémicupulari cum excipulo connivente praedita, Asci crebri subclavati 8-spori, paraphysibus ląxis claraeformibus, obvallati ; sporidia bilocularia elleptica, oblongiusabcula subveniformia constricto-didyma, primum diaphana dein badia, tandem fuligineo opaca. 
Thallus cartilagineus bullato-squamosus, ramulosus, in crustam rugoso-plicatam gyrosamque, caespitosam, pulvinatam, intestiniformem, confertus.

Obs. Differt hoc genus a Buelliis, sicut 7 h slooidtma a Lecideis.

\section{6) Cormothecium Dubenii.}

Syn. Lecidea Dubenii Fries Sum. Veg. Scand. pag. 114 ( Fide Krempel!) Lecidea Bayerrhofferi Schaer Enum. pro parte, non autem Lich. helv. n. 622 !

\section{SHORASTATIA NOV. GEX.}

Apothecia catothálama s. ex hypothallo oriunda, primum punctiformia, dein patellaria plana, tandem irregularia, plicatorugosa, angulata, centro papillata v. punctulato-urceolata, excipulo composito (fere duplici), exterius e substantia carboniosa atra undique discum obvestiens, interius albescens e substantia thalloidea in propriam mutata, praedita. Lamina proligera hypothecio agonimico imposita licheninaque instructa. Asci ventricosi creberrimi polyspori, paraphysibus mucilaginosis tenuibus granulosis, obvallati; sporidia minutissima ovoideo-elliptica diaphana, homogenea, trementia.

Thallus subfoliaceus, centro areolatus, ambitu effiguratus lacinulatus, hypothallo spongioso evanescente, praeditus.

Obs. Rationem hujus geueris probabunt ii omnes, qui bunc Lichenen diligen. ter in examen adduxerint.

\section{7) Sporastatia testudinea.}

Synon. Lecidea morio. testudinea Sch. sp. 153 exs. 227 . Biatojella el Acarospora testudinea Massal. Ricerch. pag. 13 fig. 258 - Mem. pag. 130. 
Apothecia primitus globosa clausa, dein aperta plicato-difformia patellularia, vel sublirellaeformia, excipulo composito (fere Juplici) instructa : exterius (perithecium) subcarbonaceum undique discum obvestiens, interius albescens crassiusculum cum hypothecio connivens, praedita. Lamina proligera concava v. ca* naliculata, ceracea, fusca, humecta rufescens, lichenina destituIa. Asci creberrimi polyspori, oblongo-clavati, paraphysibus conglutinatis mucilaginosis apice fuscidulis, obvallati. Sporidic mınutissima homogenea, diaphana, ovoidea, trementia (s. matu Bro. wniano exagitata.) - Thallus nullus,

\section{8 ) Sarcogyne privigna.}

Fw. Bot. Zeit. 1851 n. $43-44$ (Fide Koerb!) - Lecidea privigna Ach. Meth. pag. 49 - Lecidea simplex Borr - Pa. tellaria Wallr. - Sorcogyne corrugata v. simplex Fw.! . Acarospora immersa v. atrosanguinea Massal. Ricerch. pag. 152 exclus. Schaer. Synon. - Biatorella atrosanguinea Massal. Mim. lich. pag. 130 .

\section{9) Sarcogyne pruinosa temal.}

Syn. Lecidea-immersa v. pruinosa Schaer. Lich. hel, n. 202 - Miriosperma pruinosa Naeg. Hepp. Lich. Europ. n. 145 ! Biatorella Massal. Ricerch. 132 fig. 259.

viI. BIATOHELLA DXrks.

Apothecia hemisphaerica tuberculiformia, basi lata adnata subcarnea, laete miniata, excipulo omnino carentia, nucleo medullari, lamina proligera lichenina praedita, superiori parto ro- 
stito, composita. Asci creberrimi ublongo-clarati polyspori, pa. raphysibus tenuissimis flexuoso-capillaribus, obvallati. Sporidid elongato-linearia utrinque obtusa diaphana, sporidiolis uniseria tis globosis, foeta.

Thallus leprosus tenuissimus effusus, e meris chlorogonidiis coniogonidiisque compositus.

\section{0) Biatorella Rousselii.}

Dntrs. (Biatora Dur. et Mont. - Biatorella Massal. Ricerch. pag. 131 (ig. 257 ).

Obs. Colligitur haec speciez pulcherrima Genuae (Pro S. Siro dl strnppa) nee non Parmae (Bosco di Scola) in Ilalia.

iX. SAGIOLECHIA Nov. GEN.

Apothecia punctiformia, primum omnino in thalli substantia inclusa, dein sensim erumpentia, prominula, urceolata, tandem gyroso-plicata, inaequaliter laciniato-stellata, centro papillata, patellularia, excipulo duplici praedita. Interius conicum elevatum erumpentem e substantia thalloidea hypothallinica ac mesothallinica in propriam mutata, exterius carbonaceo-amylaceum, discum omnino perithecii ad instar obtegentem, elabentem, instructa. Lamina proligera lichenina praedita, in centro plerumque papillata, gelatinosa, hypothecio cupulari agonimico imposita. Asce clavati 8-spori, paraphysibus crassis, obvallati; sporidia elliptica fusiformia costanter diaphana tetrablastıa s. quadrilocularia.

Thallus tartareus amylaceus, areolato-contiguus, uniformis.

\section{Sagiolechia protuberans.}

Syn. Lecidea protuberans Ach. Schaer!-Bilimbia Massal. Ricerch: pag. 122 fig. 238. 
Obs. Gehus obtinet medium locum inter Gyalechs, Volvarias, Petractides, Thelotremasa elc.

\section{Х. HYMENELIA KREMP. ( CH. AUCT.)}

Apothecium subimmersum, excipulim duplex, exterius e verruca thalloidea superficiali compacta formatum, apice dehiscens, interius proprium immersum tartareum dehiscens apice connivens, basi sua thalamium amplectens. Lamina proligera discoidea subgelatinosa, lichenina praedita. Ascis multiformibus regularibus irregolaribusre clavato-stipitatis 8-sporis, paraphysibus gelatinosis evanidis, obvallatis. Sporidia diaphana oleoso-monolocularia, regularia vel irregularia v. polymorpha, sc. ovoidea, v. circularia, v. elliptica, v. angulosa, v. polygona, episporio lato cincta. Spermatocalia frequentissima papillaeformia semiimmersa, erismata fasciculata, tromodoblastia elliptica.

Thallus tartareo-amylaceus uniformis, contiguus, areolatusve, effusus.

\section{2) Hymenelia Prevostii Kremp.}

Fl. 1852 n. 2. - Synon. Hymenelia prevostii a rosea Kremp! - et b punctata (spermatocalia) - Lecidea Prevostii Schaer!

\section{3) Hymenelia hyascens Massal.}

Syn. - Himenelia prevostii v. coerulescens Kremp!- forma punctata (ipermatccalia)-Verrucaria hyascens Massal. Ricerh. pag. 177 fig. 357 - Pyrenula Ach! (Fide Schaer. Garov. etc.)

\section{4) Hymenelia coetulea Nov. Spec.}

Syn. Biátora coerulea Massal. in herb. - Legi ad saxa jurasica Prov. Veronensis in oppido Romagnano (Serbaro). 


\section{5) Hymenelia calcivora Massal.}

Syn. Lecidea calcirora Massal. Ricerch. pag. 78 fig. 158 Lecidea immersa v. calcivora Schaer! Lich. helv. n. 201 Lichen calcivorus Ehrh. (genuinus!)

\section{6) Hymenelia affinis Nov. Spec.}

Synon. Biatora affinis Massal. in herb. Legi ad saxa cretacea Prov. Veronensis, in oppido Tregnago (M. Castello).

\section{7) Hymenelia lithofroga Nov. Spec.}

Sin. Biatora lithofraga Massal. in herb. Legi in oppido Tres gnago (M. Brojo - Viacara elc. )

I1. ENCERHALOGPAPHA NOV. GEN.

Apothecia primun fere punctiformia, matricibus immersa, dein emersa plus minusve patellularia, elongatula, cylindrica, tandem multiformiter intertexta canaliculata, $v$. in globum elevatum intestiniformem spiraliter-gyrosum, aggregata: excipulo proprio, carbonaceo aterrimo, discum fulciente, praedita. Lamina proligera absque lichenina, tenuis subcilindrica. Asci rari, parvi, 8-spori, paraphysibus granulosis, mucilaginosis, inconspicuis, obvallati: sporidia elliptica primum diaphana dein fuscidula, medio constricto-didyma bilocularia nucleolata.

Thallus crustaceus uniformis, amylaceus.

\section{8 ) Eucephalographa Elisae Nor. Spee.}

Syn. Opegrapla Elisae Massal. in herb. 
Obs. Genus Graphliearum pedistinctum 1! Detexit Nob. Eusa Parount, praestans botanicae cultrix, et quidem ad rupes dolomiticas oppidi Olerii apud jam cogritas speluucas; quapropler ejus nomini dicatam speciem ruluimue.

\section{9 ) Encephalographa rubiformis Nov.Sp.}

Syn. Opegrapha ruhiformis Massal. in herb. Ad saxa jura. sica Forijulii in oppido Alloc (Pulfero) legit Sabbata Farmacopola.

XI1. LEGIOGPAPEA NOF. GEX.

Thallus proprius nullus. Apothecia parassitica punctiformia immersa, dein emerso-sessilia lecideaeformia pulvinata, tandern lirellaeformia-scutellata, gyroso-plicata, excipulo carbonaceo pro. prio aterrimo instrucla. Lamina proligera tenuis absque lichenina, subcilindrica, hypothecio pingui gonidiis impolito, impo. sita. Asci crebri 8-spori, paraphysibus crassiusculis coloratis, im. mixti; Sporidia elliptica primum diaphana homogenea, dein $2-4$ locularia, tandem tetrablastia-aplopyrenia fuligineo-fusca.

Obs. Genus dubiuin, fartasse ad liysteric referendum

\section{0) Leciographa parassitica Nov. Spec.}

Syn. Opegrapha parasitica Massal. in litt. ad divers. Legi ad sax. jurasica oppidi Tregnago, in thallo Urceolarias Cal. careae.

XIIT. KREMPLLHUBERIA NOV. GEN.

Apothecia catothalama primum punctiformia immersa, thalli. que in substantia omuino clausa, dein erumpentia lecideaeformia, tandem urceolato-sessilia, naviculari-sublirellaeformia, provecta aetate rugoso-difformia, excipulo duplici, exterius substautia 
thalloidea amulacea nonunquam obliterata, interius proprium curneo-carbonaceum, constituta ; lamina proligera pinguis gelatinosa hypothecio crasso imposita, perithecioque evanido tecta, margine proprio cincta, lichenina destituta. Asci creberrimi cylindraceo-elongati fere in stipitem attenuati, 8-spori, paraphysibus capillaribus tenerrimis creberrimisque, obvaílati, Sporidia uniserialia, tenerrima mucilaginosa, ex mera lichenina composita ( ${ }^{*}$, ovoidea, oleoso-viridula, tandem leviter fuscidula, tetrablastia-diplopyrenia.

Thallus uniformis tartareo-amylaceus effusus, nonnunquam obsoletus.

Obs. Locandum hoc genus inter Graphideas, prope Lecansctides. Dicatum Prseclaro lichenographo atque amico, D. Krempelhuber Munacensi.

\section{1) Krempelhuberia Cadubriae.}

Syn. Lecanactis Massalongi Montagn. in litter. 1854. Opegrapha Cadubriae Massal. in herb. Legi magna manu in Alpibus Cadubriae Provin. Bellunensis (Comelico) in loco dicto M. Spina ad Faros.

\section{B. ANGIOCARPI.}

IIV. SPHAERoMPHALE REICH. ( EMEND.)

Apothecium e subiculo oriundum, in thalli substantia primitus immersum, dein prominulum, conoideum, excipulo duplici praeditum: exterius e subsiantia thalloidea v.tuberculo thalloideo formatum; interius proprium corneo-carbonaceum subtus defciens, apiceque prominulum, thalamium, absque lichenina, fari-

(*) Cum jodio nallam exhibet reactionem mucilago bymenea, et tantummodo eporde, coeruico colore tingunlar. 


\section{6}

uoso-gelatinosum, servans. Asci clavati 1-2-3-spori, mucilagino granulosa, immixti, sporidia ovoideo-elliptica, tetrablastia-diplonyrenia, primum diaphana, dein badia, tandem fuligineooyaca.

Thallus cartilagineus, effusus, squamulosus foliolosus.

Ob, Differt hoc genus a Polyblitstiis, slcut Lithoiceae a Verridcarlls.

\section{2 ) Sphaeromphale Silesiaca Massal.}

Syn. Sphaeromphale thelostoma lioerb! (won Reichen.) in Litter. - Legil Koerb. ad saxa in Silesia ( Nirschberg.)

\section{BLASTODESMIA MASSAL. ( EMEND.)}

Apothecium sessile semi-immersum, excipulo simplici proprio (perithecium) carbonaceo cupuliforme subtus late deficiente, thalamiumque, absque lichenina, farinosum, servante, praeditum. Asci creberrimi 8-spori, paraphysibus mucilaginosis, obvallati, Sporidia elliptico-linearia oblonga utrinque obtusa, baculiformia 6-8-10-locularia (raro diplopyrenia) costanterque fuligineo-fusca.

Thallus hypophlaeodes.

Obs. Blastodesmiz lactea Masal. Ricerch, referenda potius est ad Polyblustias.

\section{3 ) Blastodesmia nitida Massal.}

Ricerch. pag. 180 fig. 368 a, b.

$$
\text { XVI. ARTHOPYRENIA MASSAL. (BMEND.) }
$$

Apothecium solitarium v. aggregatum superficiare v. semi inmersum hemisphaericum; excipulum simplex corneo-carbona- 
ceum, absque lichenina, thalamium farinoso-gelatinosum, servans, frimum clausum dein vix apice pertusum, tandem nucleo erumpente subdepressum, fatiscentem. Asci clavati 8-spori, mucilago hymenea rel paraphysibus mucilaginosis vix conspicuis obsoletisve, consiti. Sporidia costanter diaphana, ovoideo-elliptica $\therefore$ elliptico-clavaeformia, medio constricto-didyma, inaequaliter divisa, 2-6-10-locularia, raro diplopyrenia.

Thallus uniformis effusus plerumque hypophlaeodes.

Obs. Huc referendae omies Arthopyreniae Massal. Ricerch. excepit. n. 308-3to.

XVII. ACROCORDIA NUT. GEN

Apothecium semiimmersum v. innatum, conicum v.hemisphaericum, excipulo simplici corneo-carbonaceo subtus deficiente, praeditum, thalamiumque gelatinosum, absque lichenina, servante. Asci cylindraceo-oblongi creberrimi 8 -spori, paraphysibus distinctis capillaribus, obvallati; sporidia uniseriata ovoidea, oleoso-viridula, medio leniter constricta subdidyma, constanter bilocularia, aequaliter divisa, semper diaphana.

Thallus tartareo-farinosus vel stuppeus aracnoideusque uniformis, elfusus.

\section{4) Acrocordia gemmata.}

. Syn. Arthopyrenia Massal. Ricerch. I. 308 pag. 166.

\section{5 ) Acrocordia Garovagli.}

Syn. Arthopyrenia epipolaea Massal. Ricerch. loc. cit. $\mathbf{n}$. 510 escl. syn. (si n. 99 Hepp. Flech. Europ. sit genuina species.) - Verrucaria epipolaea et conoidea Garov! nec Ach. nec fries. 
Apothecia solitaria catothalama s. ex hypothallo oriunda, primum punctiformia omnino clausa, in thalli tuberculis, inclusa, dein emergentia urceolata, tandem fere patellaria elevata: excipulum duplex, exterius crassum conico-truncatum e tuberculo thalloideo unico formatum, interius proprium carbonaceum om. nino clausum subtusqne deficiens, thalamiumque farinosum, $a b$ sque lichenina, tandem irregulariter fatiscens, servans. Asci clavato-saccati octospori, paraphysibus granulosis crassiusculis, mucilaginosis, obvallati; sporidia ovoidea diaphana homogenea, intus nubiloso-granulosa. Spermatocalia minula pyriformia, erismata articulata, tromodoblastia lineari-elliptica.

Thallus crustosus, cartilaginosus, areolatus, ex areolis planis v. tuberculiformibus discretis, elevatisque coustitutus, hypothallo nigro byssoideo effuso, insidentibus.

\section{6) Mosigia gibbosa Fr.}

Syn. Sagedia gibbosa Fries lich. Eur. - Pyrenula gibbosa Ach. - Pyrenothea gibbosa Massal. Ricerch. pag. 152 fig. 298 (tromodoblastia) - Lecanora Grimselana Massal. Ricerch. pag. 1 fig. 2- Parmelia badia v. dispersa Schaer ! Verrucaria Grimselana Hepp. Flech. Eur. n. 225.

\section{tencoson:ce}

\section{APPENDIX}

Quum tempus in praesenti desit ut aliqud adjiciam, adnotabo per transennam, aliquot emendationes, quae faciandae sunt meis opusculis lichenologicis, et nomina tantum recensebo nonnullarum lichenum specierum, quas novissime detexi, praeterito autumno, in Alpis Venetis. - 
27) Callopisma Dalmaticum Nov. Spec. Legit ad saxa in Dalmatia Cl. D. Lanza.

\section{8 ) Pannaria Gayana.}

Syn. Parmelia Montag! - Pannaria chilensis Fèe, Massal.' Ricerch. pag. 111)

\section{9 ) Rinodina Lecanorina.}

Syn. Mischoblastia Massal. Ricerch. pag. 41 fig. 70.

\section{3o) Rinodina oxidata.}

Syn. Mischoblastia Massal. Ricerch. pag. 42 fig. 73.

\section{1) Lecidea thrombioides.}

Syn. Verrucaria thrombioides Massal. Mem. lich. pag. 1/4 fig. 173 fab. 26.

\section{2 ) Lecidea confluens v. tumida.}

Syn. Lecidea tumida Massal. Ricerch. pag. 68 fig. 127 - Biatora confluens v. vulgaris Hepp Flech. Eur. n. 125 ( affinis, quoad thallum, sed apothecia in v. tumida triplo majora).

\section{3 ) Catillaria Philippea.}

Syn. Lecidea Montag ! - Lecidea lutosa Montag. (non Ach.) Catillaria Massal. Ricerch. pag. 79 fig. 159.

\section{4) Catillaria Ricasolii.}

Syn. Abrothallus Massal. Ricerch. pag. 89 fig. 183.

\section{5 ) Catillaria arthonioides.}

Syn. Lecidea Fèe - Abrothallus Massal. Ricerch. pag. 89 fig. 184 .

\section{6 ) Catillaria (Buellia?) exilis.}

Syn. Abrothallus Massal. Ricerch. pag. 88 fig. 182 - Lecidea synothea v. exilis Flork! 


\section{7) Biatora Boutellii.}

Syn. Lecanora dein Parmelia Desmaz! Plan. Crypt. 11. 1595. Lecidea rosella Mer.

\section{8 ) Buellia leptocline.}

Syn. Buellia saxorum Massal. Ricerch. pag. 82 fig. 169. (Cum Lecidea Spuria Hepp. Fl. Europ. nihil prorsus commune habet!) - Lecidea Ieptocline Fw. ! Fide Koerb.

\section{9) Buellia (Catolechia?) scabrosa.}

Syn. Lecidea scabrosa Ach. Meth.

\section{0) Diploicia Cacuminum Nov. Spec.}

Obs. Proxima, sed diversissima Psorae nimbosae Hepp (Diploicia nimbosa Massal.) Fl. Europ. 11. 82 - Mea Diploicia muscorum Ricerch. nihul commune habet cum Lecidea insigni ab Heppio evulgata.) - Iu summis M. Spina alpium Cadıbriae. Legi.

\section{1) Diploicia nimbosa.}

Syn. Psora nimbosa IIepp. Fl. Europ. n. 82! Parmelia amniocola var. nimbosa Schaer. Enum.

\section{2.) Diploicia Trevisanii.}

Syn. Psora Trevisanii Hepp. Fl. Europ. n. 80 !

43 ) Biatora cadubriae Nov. Spec.

Legi in Apibus Cadubriae in loco dicto Col della Favola ad truncos Pinorum propre pagum Auruntium.

44 ) Arthrosporum accline.

Syn. Lecidea acclinis Fw! Fide Koerb. - Arthrosporum populorum Massal. Mem. lich. pag. 128 fig. 199 tab. 29.

45) Psora lugubris.

Syn. Lecidea lugubris Fries! Fide Krempel. 
46 ) Haematomma elatinum.

Syn. Loxospora elatina Massal. Ricerch. pag. 138 fig. 272 sporae immaturae.

\section{7) Petractis Friesii.}

Syn. Gyalecta discolor Fw! - Gyalecta Friesi Fw - Fide Koerb.

\section{$48)$ Gyalecta? abstrusa.}

Syn. Lecidea cornea v. abstrusa Schaer. Enum. - Gyalecla Wahlembergii v. truncigena Schleich! Fide Koerl.

\section{9) Rocella intricata Montagn !}

Syn. Rocella Flaccilla Massal. Mem. pag. 69 tab. 12 fig. 77. 5o) Endocarpon Moulinsi Mont.

Obs. Optima ac formosissima species!

\section{1) Endocarpon miniatum v. exaspera-}

\section{tum.}

Syn. Endocorpon Moulinsii Massal. Ricerch. pag. 186 - Schaer Enum. (non Montag.) - Specimem ab Heppio evulgatum Fl. Eur. 11. 218 nihil commune habet cum End. Moulinsi a Schaererio edito!, quapropter omuia synon. emendauda.

\section{Dermatocarpon clopimum.}

Syn. Dermatocarpon glomeruliferum Massal. Mem. Lich. pag. 141 - Thelotrema clopimum Hepp. Exs. Fl. Eur. n. 101 esclus. syn. Schaer. et Massal (si vera sit Heppii species!) Thelotrema Sclsaereri Hepp. exs. Fl. Eur. n. 100 exclus. syn. Trevis. et Montagn!

\section{3 ) Dermatocarpon protuberans.}

Syn. Paraphysorma protuberans Massal. Ricerch. pag. 117 fig. 228 - Lecanora cervina v. protuberans Schaer!-('The- 
lotrema clopimum v. porphyrium Hepp. n. 102-Verrucaria porphyria Meyer. - Pyrenula Schaer. Enum.??)

\section{4) Dermatocarpon Ambrosianum.}

Syn. Paraphysorma Ambrosianus Massal. Mem. Lich. pag. 156 fig. 162 tab. 24.

\section{5) Dacampia Engeliam.}

Syn. Sagedia Engeliam Saut! in Rabeh. Bot. centralb. 1846 n. 20 Fide Krempel!

\section{6) Omphalaria nummolaria Montag.} Syn. Collema Duf. - Collema helvelloideum Dntrs! Garov! (non Ach.) Omphalaria? helvelloidea Massal. Mem. lich. pag. 90 fig. 108 tab. 17.

\section{$57)$ Porina tenebricosa Nov. Spec.}

Syn. Porina muscorum v. tenebricosa Massal. in herb. Legi ad truncos Pinorum in Alpibus Cadubriae, in tenebricosis, in loco dicto Tre Ponti prope Auruntium.

\section{8 ) Verrucaria Auruntii Nov. Spec.}

Obs. Proxima Verr. aethiobolae Wal. et V. Flotowianae Heppi, sed satis diversa. Legi in Alpibus Cadubriae prope pagum Auruutium ad rupes dolomiticas.

\section{9 ) Verrucaria confluens Nov. Spec.}

Obs. Proxima n. 93 Hepp. Fl. Europ. sel diversa. - Legi ad saxa arenaria corrupta Prov. Veronensis, in oppido Tregnago (M. Castello).

6o) Verrucaria (amphoridium?) Dolomitica Nov. Spec.

Syn. Verrucaria epipolaea v. albida Garov! forma. Legi all saxa Dolomitica Prov. Veronensis, in oppido Giazza, nec non ad Larium lacum Garov! 
6ı) Lithoicea Funkii Massal. Mem. Lich. pag. 143.

Syn. Pyrenula Funkii Spreng. Funk. Crypt. Germ. n. 658!Verrucaria Leigthonii Hepp! FI. Eur. n. 95 syn. castig. Verrucaria viridula Massal. (non Ach!) Ricerch. pag. 171 fig. 343. - Lithoicea Massal. Mem. pag. 142.

62 ) Lithoicea? laevata.

Syn. Verrucaria laevata Ach. Schaer. Enum. 217! Fide Koerb.

\section{3 ) Lithoicea tectorum Nov. Spec.}

Syn. Verrucaria tectorum Massal. in litt. ad divers. - Legi ad tecta Provin. Veronensis, potissimum in oppido Tregnago.

$64)$ Amphoridium uberinum Nov. Spec. Obs. Legi ad saxa arenaria jurasica Prov. Veronensis in oppido Tregnago (Fontanine).

65) Amphoridium (Nov. Genus?) amylaceum Nov. Spec.

Syn. Verrucaria amylacea Massal. in herb. Legi ad saxa eocenica Prov. Veronen. in umbrosis M. Purga oppidi Velo.

66) Sagedia Oleriana Nov. Spec.

obs. Legi ad saxa dolomitica Prov. Vicetinae, prope speluncas oppidi Olerii.

67) Pyrenula Schaererii.

Syn. Verrucaria Schaererii $F_{w}$ ! Fide Koerb. - Pyrenula quercus Massal. Mem. lich. pag. 138 fig. 170 tab. 25.

68) Polyblastia ventosa Nov. Spec.

Syn. Verrucaria ventosa Massal. in herb. Legi ad saxa cretacea Pr. Veronensis in ventosis oppidi Tregnago (M. Viacara, I1. Brojo, Precastio, Gardon etc.) 
24

\section{9) Polyblastia dermatodes Nov. Spec.}

Syn. Verrucaria Massal. in herb. Legi ad saxa eocenica corrupta Prov. Veronensis, in umbrosis oppidi Velo, M. Purga. 7o) Polyblastia rupifraga Nov. Spec. Syn. Verrucaria Massal. in muscr. Legi cum priore.

\section{I) Polyblastia Lariana Massal.}

Syn. Verrucaria rupestris v. calciseda Garov! (non Schaer!). Legit circa Larium Lacum ad saxa calcareo-bituminosa Clar. Prof. Garov!

\section{2) Arthopyrenia cinerescens Nov. Spec.}

obs. Legit ad Fraxinos prope Bassanum (Campo di Rimano) Pharmacopola Fs. Beltramini.

\section{3 ) Enchylium Rubbianum Nov. Spec.}

Syo. Collema monocarpum Duf.?? Legit ad saxa arenacea M. Rubbii in Prov. Vicetina, Fr. Bell ramini. 


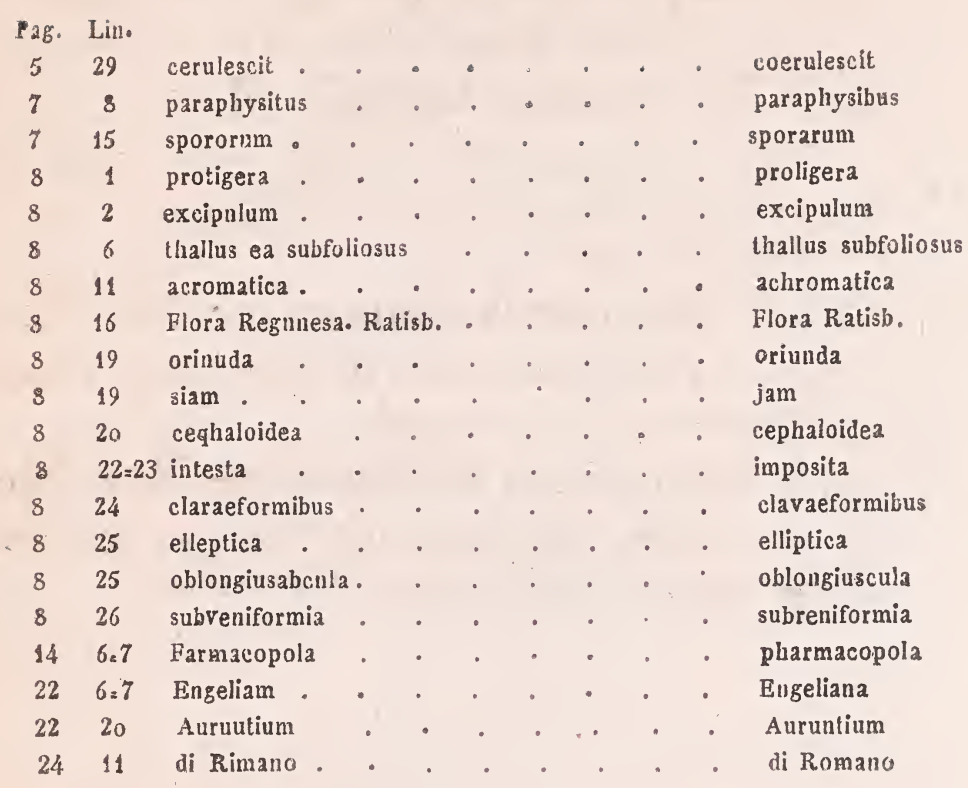






\section{MONITUR:}

Apud bibliopolan A. F. Münster, Venetiis aut Veronae, sunt venalia sequentia opuscula lichenographica, ejusdem auctoris:

1) Nota sulla Lecidea Bolcana di Ciro Pollini. Veronze = 1551 = 8 pag.

2) Animadversio in Lecideam Bolcanam Cyrii Pollinii. Ratisbonae o 1851 . 6 pag. o vel Bononiae 1852.

$3)$ Sporodictyon norum Lichenum Genus. Ratisbonae 1852 . 3 pag. 1 tab. lith. = Vel Bononiae absque tabula.

4) Amphoridium norum Lichenum genus. Ratisbonae 1952, vel Venetiis 1853, 12 pag. 1, tab. color. lith.

5) Sui generi Dirina e Dirinopsis della Famiglia dei Licheni. Vindobonae 1952, 18 pag. 4 tab. lithog.

6) Synopsis Lichenum blasteniospororum.

Ratisbonae 1852, 16 pag.

7 ) Ricerche sull' antonomia dei licheni crostosi.

Veronae $1852=221 \mathrm{pag} .64 \mathrm{tab}$. lithoz. cuns 400 figur.

8.) Memorie Lichenografiche.

Veronae 1853, par. 131, tab. 29, cum 2no fig。 lithogr.

$9)$ Monografia dei Licheni blasteniospori.

Venetiis 1853, pag. 131, tab. 6 dupl. 36 fig. lithogr.

10) Summa animadversionum in duos postremos fasciculos Lichenum Helveticorum editos a L. E. Schaerer.

Veronae 1853 , pag. 13.

11) Osservazioni sopra i due ultimi fascicoli di Licheni pubblicati dallo Schaerer.

Bononiae 1853, pag. 26, tab. 2 lithogr.

12 ) Alcuni generi di Licheni nuovamente limitati e descritti. Veronae 1853 , pag. 14.

13) Sulla Lecidea Hookeri di Schaerer.

Veronae 1853, pag. 9, tab. 1 lithogr = omnia in $80^{\circ}$ 